\title{
pharma-kritik
}

AZA $9500 \mathrm{Wil}$

ISSN 1010-5409

Jahrgang 24

Nummer 14/2002

\section{Nebenwirkungen aktuell}

\section{ERYTHROPOETIN}

Rekombinantes Erythropoetin, ein Glykoprotein, stimuliert die Erythropoese und wird zur Behandlung von Anämien eingesetzt, die mit chronischer Niereninsuffizienz, einem Tumorleiden oder einer Zytostatikatherapie in Zusammenhang stehen. Ferner wird das Hormon vor Operationen verwendet, um Eigenblutspenden vorzubereiten oder den Bedarf an Fremdblutspenden zu reduzieren. Es gibt in der Schweiz drei verschiedene Erythropoetin-Präparate: Epoetin alfa, Epoetin beta und Darbepoetin; sie unterscheiden sich im Glykosylierungsmuster, sind in ihren pharmakologischen Wirkungen aber identisch.

Übersichten zu verschiedenen Einsatzgebieten:

Valderrabano F. Kidney Int 1996; 50: 1373-91

Cazzola M et al. Blood 1997; 89: 4248-67

Duhrsen U. Drugs 2002; 62: 2013-23

Crosby E. Am J Ther 2002 ; 9: 371-6

Markennamen: Epoetin alfa: Eprex ${ }^{\circledR}$, Epoetin beta: Recormon $^{\circledR}$, Darbepoetin alfa: Aranesp ${ }^{\circledR}$

\section{Erythroblastopenie}

Es werden 13 Personen im Alter von 22 bis 85 Jahren beschrieben, die wegen diverser Nierenkrankheiten an einer chronischen Niereninsuffizienz litten und bei denen unter einer Therapie mit Erythropoetin eine Erythroblastopenie («pure red cell aplasia») auftrat. Bei elf Personen wurde eine Hämodialyse durchgeführt, bei einer eine Peritonealdialyse und bei einer fand keine Dialyse statt. Alle hatten Erythropoetin subkutan erhalten. Die Dauer von Beginn der Behandlung bis zum Auftreten einer schweren, nicht mehr auf Erythropoetin reagierenden Anämie lag zwischen 3 und 67 Monaten.

Acht Personen hatten nur Epoetin alfa verwendet und eine Person nur Epoetin beta; bei vier Personen waren beide Präparate eingesetzt worden, wobei es in diesen Fällen Epoetin alfa war, das in den Monaten unmittelbar vor der Manifestation der Erythroblastopenie verordnet war. Die hämatologische Dia- gnose erfolgte in 12 Fällen via Knochenmarkspunktion, in einem Fall, indem sich im peripheren Blut keine Retikulozyten nachweisen liessen.

Um einen Tumor oder viralen Infekt auszuschliessen, wurden bei allen Betroffenen eine Computertomografie von Thorax und Abdomen sowie serologische Untersuchungen durchgeführt. Alle 13 Personen wiesen Antikörper gegenüber Erythropoetin auf. Bei 10 Personen war im Serum kein Erythropoetin nachweisbar. In vitro vermochten die Seren die Erythropoese in Knochenmarkskulturen von Gesunden zu hemmen. Nachdem Erythropoetin abgesetzt und in den meisten Fällen eine immunsuppressive Therapie begonnen worden war, erholte sich die Erythropoese bei einigen Betroffenen wieder; 3 blieben aber bis zum Abschluss der Beobachtungsperiode transfusionsbedürftig.

Casadevall N et al. N Engl J Med 2002; 346: 469-75

\section{Bei Niereninsuffizienz nicht subkutan verabreichen!}

Wie mehrere Arzneimittelbehörden mitgeteilt haben, sind bis Ende September 2002 weltweit 165 Fälle von Erythroblastopenie unter Epoetin alfa erfasst worden, bei denen man die Diagnose mit einer Knochenmarkspunktion bestätigt hatte; in 112 Fällen ist dokumentiert, dass Antikörper gegen Erythropoetin vorhanden waren. In praktisch allen Fällen handelte es sich um

\author{
Stichwortverzeichnis zu dieser Ausgabe \\ Darbepoetin alfa \\ Demyelinisierungen (TNF- $\alpha-$ Blocker) \\ Epoetin alfa/beta \\ Erythroblastopenie (Erythropoetin) \\ Erythropoetin \\ Etanercept \\ Glukoseintoleranz (Risperidon) \\ Infliximab \\ Kardiale Arrhythmien (Risperidon) \\ Lymphoproliferative Erkrankungen (TNF- $\alpha$-Blocker) \\ Risperidon \\ TNF- $\alpha$-Blocker \\ Zerebrovaskuläre Ereignisse (Risperidon)
}

\section{Texte dieser Ausgabe}

zusammengestellt und kommentiert

von E. Gysling (EG) und UP. Masche (UM) 
Personen mit chronischer Niereninsuffizienz, die Epoetin alfa subkutan erhalten hatten.

Deshalb haben die Behörden in den EU-Ländern und in der Schweiz angeordnet, dass Epoetin alfa bei chronischer Niereninsuffizienz nicht mehr subkutan, sondern nur noch intravenös verabreicht werden darf (andere Erythropoetin-Indikationen sind von dieser Einschränkung nicht betroffen). Bei Personen, bei denen unter Erythropoetin eine Erythroblastopenie aufgetreten ist, dürfen keine Erythropoetin-Derivate mehr verschrieben werden. Eine Therapie mit Erythropoetin muss durch regelmässige Bestimmungen der Retikulozytenzahl überwacht werden, um eine fehlende Wirkung von Erythropoetin bzw. eine Erythroblastopenie möglichst früh zu erkennen.

Informationen von Swissmedic:

http://www.swissmedic.ch/cgi/news/index.asp?sitetype=

laien\&news $\mathrm{id}=2588$

Informationen der britischen Arzneimittelbehörde:

http://www.info.doh.gov.uk/doh/embroadcast.nsf/vw

DiscussionAll/35595645F6304BFA80256C8D00456DCD

Es fällt auf, dass fast nur im Zusammenhang mit Epoetin alfa über die Erythroblastopenie berichtet wurde. Dies bedeutet wohl nicht, dass Epoetin alfa das gefährlichste ErythropoetinDerivat wäre, sondern dürfte als Ausdruck unterschiedlicher Verschreibungsfrequenzen anzusehen sein. Es erscheint deshalb im Augenblick sinnvoll, die für Epoetin alfa formulierten Vorsichtsmassnahmen auch auf die beiden anderen Substanzen zu beziehen. Das Beispiel von Erythropoetin lehrt einen wieder, dass bei Medikamenten auch nach jahrelanger Verwendung neue Probleme bekannt werden können und man gegenüber möglichen Nebenwirkungen nie die Wachsamkeit verlieren darf. (UM)

\section{TNF- $\alpha-B L O C K E R$}

Die beiden TNF- $\alpha$-Blocker Etanercept und Infliximab hemmen den Tumornekrosefaktor alpha, ein proinflammatorisches Zytokin, und werden zur Behandlung der rheumatoiden Arthritis eingesetzt, wenn andere Basistherapien nicht genügend helfen. Infliximab wird zudem bei schweren Fällen von Morbus Crohn verwendet.

Informationen zu Etanercept und Infliximab :

Masche UP. pharma-kritik 2002; 24: 9-12

Luong BT et al. Ann Pharmacother 2000; 34: 743-60

Jobanputra P et al. Health Technol Assess 2002; 6: 1-110

Markennamen: Etanercept: Enbrel ${ }^{\circledR}$, Infliximab: Remicade $^{\circledR}$

\section{Demyelinisierungen/Veränderungen der weissen Substanz}

Ein 48jähriger Mann war wegen einer rheumatoiden Arthritis 4 Monate lang mit Etanercept (2mal $25 \mathrm{mg} /$ Woche) behandelt worden, als Fieber, Verwirrtheit, eine leichte linksseitige Fazialisparese und eine Ataxie auftraten. In der Annahme eines Infektes wurde eine Behandlung mit Ceftriaxon $\left(\right.$ Rocephin $\left.^{\circledR}\right)$, Metronidazol (Flagyl ${ }^{\circledR}$ u.a.), Aciclovir (Zovirax ${ }^{\circledR}$ u.a.) und Methylprednisolon $\left(\right.$ Solu-Medrol ${ }^{\mathbb{R}}$ ) begonnen.
Nachdem man in der Kernspintomografie (MRI) periventrikulär und subkortikal eine erhöhte Signalintensität in den weissen Arealen festgestellt hatte, wurde der Patient in ein Zentrumsspital verlegt. Bei Eintritt hatte er $38,4^{\circ} \mathrm{C}$ Fieber und reagierte lediglich auf Schmerzreize; am folgenden Tag wurde er bewusstlos. Im Liquor konnte eine erhöhte Eiweisskonzentration von $79 \mathrm{mg} / \mathrm{dl}$ nachgewiesen werden. Das EEG zeigte eine allgemeine Verlangsamung, die Angiografie normale Hirngefässe. In der nochmals anfertigten Kernspintomografie fand sich eine Zunahme des abnormen Befundes in der weissen Substanz. Das histologische Bild der Gehirnbiopsie war am ehesten mit einer Leukenzephalopathie vereinbar.

Nach einer fünftägigen Steroidbehandlung besserte sich der Zustand allmählich. Bei der Nachkontrolle nach sechs Wochen war nur noch die Fazialisparese zu beobachten.

In der Nebenwirkungs-Datenbank der amerikanischen Arzneimittelbehörde (FDA) wurden 19 weitere Personen identifiziert, bei denen sich unter TNF- $\alpha$-Blockern neurologische Symptome entwickelt hatten (17 hatten Etanercept, zwei Infliximab erhalten). Am häufigsten waren Parästhesien, Optikusneuritis und Sehstörungen, Verwirrtheit, Gangstörungen, Apraxie, Fazialisparese und Guillain-Barré-Syndrom. Die Behandlungsdauer bis zum Auftreten der neurologischen Symptome lag zwischen einer Woche und 15 Monaten. Die meisten der Betroffenen zeigten in den MRI-Bildern Befunde, die an demyeliniserende Prozesse erinnerten (also anders als der oben beschriebene Patient). Vier Personen hatten bereits früher MSähnliche Symptome erlebt, so dass die jetzigen Beschwerden als ein Wiederaufflackern angesehen wurden. Bei den meisten der Betroffenen besserte sich der Zustand teilweise oder vollständig, nachdem man den TNF- $\alpha$-Blocker abgesetzt hatte; ein Patient zeigte einen positiven «Rechallenge», indem nach erneuter Therapie mit Etanercept die neurologischen Beschwerden wiederkehrten.

Mohan N et al. Arthritis Rheum 2001; 44: 2862-9

\section{Lymphoproliferative Erkrankungen}

In einer Fallserie, ebenfalls anhand von FDA-Nebenwirkungsdaten zusammengestellt, wird über 26 Personen berichtet, bei denen unter einer Behandlung mit TNF- $\alpha$-Blockern eine lymphoproliferative Erkrankung diagnostiziert wurde. 18 Meldungen betrafen Etanercept, 8 Meldungen Infliximab. Bei den lymphoproliferativen Erkrankungen handelte es sich vor allem um Non-Hodgkin-Lymphome; in 4 Fällen war es ein HodgkinLymphom, in einem ein Thymom. Einige der Betroffenen waren neben Etanercept und Infliximab noch mit anderen immunsupprimierenden Medikamenten behandelt worden. Bei dem Patienten mit einem Thymom schrumpfte der Tumor, nachdem man Etanercept gestoppt hatte. Bei einem anderen Patienten nahmen nach Absetzen von Infliximab die axillären Lymphknotenschwellungen deutlich ab. Auffallend war, dass die Latenzzeit vom Beginn der Behandlung bis zur Diagnose des Tumors im Durchschnitt kurz war (mediane Dauer 6 bzw. 8 Wochen) - was zum Beispiel auch für Lymphome typisch ist, die sich nach Organtransplantationen entwickeln. Jene Lymphome sind in den meisten Fällen mit dem Epstein-BarrVirus assoziiert, so dass dieser Zusammenhang bei den TNF$\alpha$-Blockern ebenfalls erwogen werden muss.

Brown SL et al. Arthritis Rheum 2002; 46: 3151-8 
Fast gleichzeitig mit der Einführung der TNF- $\alpha$-Blocker wurden Fälle beschrieben, die darauf hinwiesen, dass unter diesen Medikamenten eine Tuberkulose oder andere opportunistische Infekte auftreten bzw. reaktiviert werden können. Der Bericht zu den Lymphomen unterstreicht, dass die immunsupprimierenden Eigenschaften der TNF- $\alpha$-Blocker nicht unterschätzt werden dürfen. Dass die Blockade von TNF- $\alpha$ einen Eingriff in ein komplexes System bedeutet, dessen langfristige Folgen noch kaum bekannt sind und dem man bei allem Enthusiasmus Respekt zollen sollte, zeigen auch die Demyelinisierungs-Fälle. (UM)

\section{RISPERIDON}

Im Vergleich mit den anderen atypischen Neuroleptika ist Risperidon für ein grösseres Indikationsspektrum zugelassen. Neben der Schizophrenie gelten auch Wahnvorstellungen und Aggressivität bei Dementen sowie manische Phasen bei bipolaren Störungen als Indikation.

Neuere Übersichten zu Risperidon:

Kennedy E et al. Cochrane Database Syst Rev 2002;

(2): CD000440

Schweitzer L. Int Clin Psychopharmacol 2001; 16: 1-19

Bhana N, Spencer CM. Drugs Aging 2000; 16: 451-71

Markenname: Risperidon: Risperdal ${ }^{\circledR}$

\section{Zerebrovaskuläre Ereignisse}

In einer australischen Studie wurde Risperidon bei dementen Kranken mit Verhaltensstörungen gegen Placebo getestet. Von 167 Personen, die Risperidon erhielten, hatten 15 ein zerebrovaskuläres Ereignis (Schlaganfall oder transitorische ischämische Attacken). Von 170 Personen, die Placebo erhielten, erlitten nur 3 ein zerebrovaskuläres Ereignis.

Zusammenfassend wird über 4 kontrollierte Studien bei insgesamt 1200 dementen Kranken berichtet, die während 1 bis 3 Monaten Risperidon oder Placebo erhielten. Unter Risperidon waren zerebrovaskuläre Ereignisse doppelt so häufig wie unter Placebo. Acht weitere Fälle von zerebrovaskulären Ereignissen wurden in offenen Studien beobachtet, die 454 mit Risperidon Behandelte umfassten. Insgesamt sind mindestens 37 Fälle von zerebrovaskulären Komplikationen bekannt; bei 16 davon kam es zum Tod.

Wooltorton E. Can Med Assoc J 2002; 167: 1269-70

\section{Kardiale Arrhythmien / plötzlicher Herztod}

Eine 34jährige Frau ohne kardiale Anamnese wurde wegen einer Exazerbation einer chronischen Schizophrenie neu mit Risperidon behandelt. Da eine orthostatische Hypotonie auftrat, wurde die Dosis nicht über 2 mal täglich $2 \mathrm{mg}$ gesteigert. Am fünften Tag dieser Behandlung hatte die Patientin einen Kreislaufstillstand. Ein EKG zeigte ein auf $480 \mathrm{msec}$ verlängertes QTc-Intervall. Die Wiederbelebungsmassnahmen blieben erfolglos, schliesslich trat eine Asystole ein. Die Autoren des Berichtes stellen fest, dass Risperidon als Ursache zum mindesten nicht ausgeschlossen werden kann.

Ravin DS, Levenson JW. Ann Pharmacother 1997; 31: 867-70
In einer Kohortenstudie wurde unter Verwendung der Verschreibungsdaten in 3 amerikanischen Medicaid-Programmen untersucht, wie häufig zwischen 1993 und 1996 ein Herzstillstand oder eine Kammerarrhythmie bei behandelten Schizophreniekranken bzw. bei nicht-schizophrenen Kontrollpersonen vorgekommen war. Die für die Behandlung der Schizophrenie verwendeten Neuroleptika waren: Clozapin $\left(\right.$ Leponex $\left.^{\circledR}\right)$, Haloperidol (Haldol ${ }^{\circledR}$ u.a.), Risperidon und Thioridazin $\left(\right.$ Melleril $\left.^{\mathbb{R}}\right)$. Im Vergleich mit den nicht-schizophrenen Kranken hatten Personen mit einer Schizophrenie ungefähr ein zwei- bis dreimal grösseres Risiko eines Herzstillstands oder einer ventrikulären Arrhythmie. Am grössten war das kardiale Risiko unter Risperidon; gegenüber den Kontrollpersonen betrug das nach Alter und anderen Variablen korrigierte relative Risiko 3,1, gegenüber den mit Haloperidol behandelten Schizophrenen 1,5. Die Studienverantwortlichen vermuten, es handle sich in Bezug auf Risperidon um ein zufälliges oder durch «Confounding» erklärbares Resultat, empfehlen jedoch weitere Untersuchungen.

Hennessey S et al. Br Med J 2002; 325: 1070-4

\section{Glukoseintoleranz / Diabetes}

In der Nebenwirkungs-Datenbank der WHO wurden alle Berichte identifiziert, die auf eine Glukoseintoleranz infolge von Clozapin, Olanzapin (Zyprexa $\left.{ }^{\circledR}\right)$ und Risperidon hinweisen. Zum Vergleich wurden in gleicher Weise auch die Berichte zu Haloperidol und Chlorpromazin (Chlorazin ${ }^{\circledR}$ ) untersucht. Es ergab sich, dass Clozapin, Olanzapin und Risperidon signifikant mit einer Glukoseintoleranz assoziiert sind, während dies für Haloperidol und Chlorpromazin nicht zutrifft. Eine Gewichtszunahme und die gleichzeitige Verabreichung von Zytochromhemmern wie die Serotoninwiederaufnahmehemmer oder Valproinsäure (z.B. Convulex ${ }^{\circledR}$ ) gehören zu den Faktoren, die das Auftreten einer Glukoseintoleranz begünstigen.

Hedenmalm K et al. Drug Saf 2002; 25: 1107-16

Die britische «General Practice Research Database» diente als Grundlage einer Fall-Kontroll-Studie, in der nach dem Zusammenhang zwischen Olanzapin oder Risperidon und dem Auftreten eines Diabetes mellitus gesucht wurde. Personen, die mit Olanzapin behandelt wurden, hatten ein signifikant erhöhtes Diabetesrisiko. Unter Risperidon war das Diabetesrisiko ebenfalls erhöht, jedoch nicht in signifikantem Ausmass.

Koro CE et al. Br Med J 2002; 325: 243-7

Aus der Literatur gewinnt man den Eindruck, Risperidon stehe gewissermassen zwischen den konventionellen und den «eigentlich» atypischen Neuroleptika. Die Substanz scheint weniger extrapyramidale Probleme als Haloperidol zu verursachen, aber mehr als z.B. Clozapin. Risperidon führt seltener zu starker Gewichtszunahme und Glukosestoffwechselstörungen als z.B. Olanzapin, aber ganz frei von dieser Problematik ist es auch nicht.

Was die hier beschriebenen kardialen und zerebrovaskulären Komplikationen von Risperidon anbelangt, sind jedoch noch einige Fragen offen. Für die Herzrhythmusstörungen ist wohl meistens eine - nicht selten durch eine Arzneimittelinteraktion verursachte - Verlängerung des QTc-Intervalls ausschlaggebend. (EG) 


\section{Nebenwirkungs-Index 1998-2002}

In diesem Index finden sich alle Beiträge, die in den speziellen Nebenwirkungs-Nummern der letzten fünf Jahre erschienen sind. Alle diese Texte finden Sie auch (via die Suchfunktion unserer Website www.infomed.org) im Internet. Dort werden in den «Bad Drug News» zudem zusätzliche aktuelle Nebenwirkungs-Informationen angeboten.

Alteplase (Anaphylaxie) 1999; 21: 55

Alteplase (Hirnblutungen) 1999; 21:54

Amfebutamon (Allergie) 2001; 23: 13

Amfebutamon (Herzrhythmusstörungen) 2001; 23: 13

Amfebutamon (Überdosis) 2001; 23: 13

Astemizol (Herzrhythmusstörungen) 1999; 21: 15

Atypische Neuroleptika (Gewichtszunahme) 2000; 22: 55

Bupropion (Allergie) 2001; 23: 13

Bupropion (Herzrhythmusstörungen) 2001; 23: 13

Bupropion (Überdosis) 2001; 23: 13

Celecoxib (Magen-Darmprobleme) 2000; 22: 15

Celecoxib (Magen-Darmprobleme) 2002; 24: 14

Celecoxib (Nephrotoxizität) 2000; 22: 15

Celecoxib (Pankreatitis) 2000; 22: 16

Cetirizin (Sedation) 1999; 21: 56

Cisaprid (erhöhte Alkoholspiegel) 1998; 20: 19

Cisaprid (Herzrhythmusstörungen) 1998; 20: 18

Clopidogrel (Arthritis) 1999; 21: 53

Clopidogrel (Glomerulonephritis) 1999; 21: 54

Clopidogrel (Purpura) 1999; 21: 53

Clozapin (Diabetes mellitus) 2000; 22: 55

Co-Amoxiclav (aseptische Meningitis) 1999; 21: 16

Co-Amoxiclav (Cholestase) 1999; 21: 15

Co-Amoxiclav (Hepatotoxizität) 1999; 21: 16

Erythropoetin (Erythroblastopenie) 2002; 24: 53

Etanercept (Demyelinisierung) 2002; 24: 54

Etanercept (Lymphoproliferation) 2002; 24: 54

Glitazone (Hepatotoxizität) 2001; 23: 56

Glitazone (Herzinsuffizienz) 2001; 23: 55

Glukokortikoide (Wachstumshemmung) 1999; 21: 55

Infliximab (Demyelinisierung) 2002; 24: 54

Infliximab (Lymphoproliferation) 2002; 24: 54

Interferone (Depression) 2001; 23: 16

Interferone (Epilepsie) 2001; 23: 16

Interferone (Hyperthyreose) 2001; 23: 15

Interferone (Thyreoiditis) 2001; 23: 15

Itraconazol (Cholestase) 2001; 23: 15

Itraconazol (Herzinsuffizienz) 2001; 23: 14

Kava-Extrakte (Hepatotoxizität) 2002; 24: 13

Metformin (Hepatotoxizität) 1998; 20: 58

Metformin (Interaktionen) 1998; 20: 58

Metformin (Laktatazidose) 1998; $20: 57$

Nimesulid (Hepatotoxizität) 2002; 24: 15

Nimesulid (Oligoanurie) 2002; 24: 16

Nimesulid (Photodermatitis) 2002; 24: 16

Olanzapin (Diabetes mellitus) 2000; 22: 55

Östrogene (Brustkrebs) 2001; 23: 54

Östrogene (Endometriumkarzinom) 2001; 23: 54

Östrogene (Gallensteine) 2001; 23: 55

Östrogene (Ovarialkarzinom) 2001; 23: 54

Östrogene/Gestagene (Brustkrebs) 2002; $24: 14$

Paroxetin (Durchfall) 2001; 23: 54

Paroxetin (Entzugssyndrom) 2001; 23: 53

Paroxetin (Gewichtszunahme) 2001; 23: 54
Paroxetin (sexuelle Dysfunktion) 2001; 23: 54

Phenylpropanolamin (Hirnblutungen) 2000; 22: 54

Phenylpropanolamin (Hypertonie) 2000; 22: 54

Pioglitazon (Herzinsuffizienz) 2001; 23: 56

Quetiapin (Diabetes mellitus) 2000; 22: 56

Risperidon (Diabetes mellitus) 2000; 22: 56

Risperidon (Glukoseintoleranz) 2002; 24: 55

Risperidon (Herzrhythmusstörungen) 2002; 24: 55

Risperidon (pulmonale Akathisie) 2000; 22: 56

Risperidon (zerebrovaskuläre Probleme) 2002; 24: 55

Rofecoxib (kardiovaskuläre Reaktionen) 2000; 22: 16

Rofecoxib (kardiovaskuläres Risiko) 2002; 24: 15

Rofecoxib (Magen-Darmprobleme) 2000; 22: 15

Rofecoxib (Nephrotoxizität) 2000; 22: 15

Rosiglitazon (Hepatotoxizität) 2001; 23: 56

Rosiglitazon (Herzinsuffizienz) 2001; 23: 55

Sertindol (Bewegungsstörungen) 1998; 20: 60

Sertindol (Herzrhythmusstörungen) 1998; 20: 60

Sildenafil (Cluster-Kopfschmerzen) 1998; 20: 59

Sildenafil (Herzinfarkt) 1998; 20: 59

Sildenafil (Interaktionen) 1998; 20: 59

Sildenafil (Kammertachykardie) 1998; 20: 59

Sildenafil (Retinaveränderungen) 1998; 20: 59

Sumatriptan (Herzinfarkt) 2000; 22: 14

Sumatriptan (ischämische Kolitis) 2000; 22: 14

Sumatriptan (Nierenvenenthrombose) 2000; 22: 14

Sumatriptan (Vorhofflimmern) 2000; 22: 14

Thioridazin (Herzrhythmusstörungen) 2000; 22: 13

Ticlopidin (Phenytoin-Interaktion) 1999; 21: 14

Ticlopidin (Purpura) 1999; 21: 13

TNF- $\alpha$-Blocker (Demyelinisierung) 2002; 24: 54

TNF- $\alpha$-Blocker (Lymphoproliferation) 2002; 24: 54

Tolcapon (Hepatotoxizität) 1998; 20: 17

Tolcapon (Verwirrungszustände) 1998; 20: 17

Vigabatrin (akute Enzephalopathie) 1998; 20: 19

Vigabatrin (Depression) 1998; 20: 20

Vigabatrin (Gesichtsfeldausfälle) 1998; 20: 19

Vigabatrin (Psychosen) 1998; 20: 20

Zafirlukast (Churg-Strauss-Syndrom) 2000; 22: 53

Zafirlukast (Hepatotoxizität) 2000; 22: 53

Zolpidem (Hepatotoxizität) 1999; 21: 15

Zolpidem (Interaktionen) 1999; 21: 15

Zolpidem (Psychosen) 1999; 21: 14

Zolpidem (tödliche Überdosis) 1999; 21: 14

Diese Nummer wurde am 29. Januar 2003 redaktionell abgeschlossen.

\section{pharma-kritik}

\author{
Internet-Adresse: http://www.infomed.org
}

Herausgegeben von Etzel Gysling (Wil)

unter Mitarbeit von Renato Galeazzi (St.Gallen) und Urs A. Meyer (Basel)

Redaktionsteam: Etzel Gysling (Leitung), Urspeter Masche, Peter Ritzmann, Thomas Weissenbach

Abonnementspreis für den Jahrgang 24 (2002, 20 Ausgaben): Fr. 96.-

Infomed-Verlags-AG, Bergliweg 17, 9500 Wil

Telefon 071-910-0866, Telefax 071-910-0877, e-mail: infomed@infomed.org

Druck: Druckerei R.-P. Zehnder AG, 9500 Wil

(c) 2003 Infomed Wil. All rights reserved. 


\section{pharma-kritik}

Jahrgang 24 Nr. 14

Beilage

\section{MARKENNAMEN}

der im Text erwähnten Arzneimittel*

( $A=$ in Österreich, $D=$ in Deutschland)

\section{Aciclovir}
A: Zovirax und viele andere
D: Acic, Mapox und andere

Chlorpromazin
A: Largactil
D: Propaphenin

Darbepoetin
A: Aranesp, Nespo
D: Aranesp

Epoetin alfa
A: Erypo
D: Eprex, Erypo

\section{Epoetin beta}
A: Neorecormon u.a.
D: Neorecormon

Methylprednisolon
A: Promedrol, Solu-Medrol
D: Medrate, Urbason u.a.

\section{Metronidazol}

\section{A: Ariline, Flagyl und andere D: Arilin, Clont und andere}

* Die Liste erhebt keinen Anspruch auf Vollständigkeit. Berücksichtigt wurden in erster Linie Markennamen, die von den Schweizer Namen abweichen. 